\title{
El Grupo operativo como método participativo: poder y aprendizaje en la relación profesional
}

\author{
The Operating Group and Participative Methods: Learning and \\ Power in the Professional Relationship
}

\author{
Lorenzo García Martín \\ Universidad Complutense de Madrid \\ lorengarciamartin@gmail.com
}

Recibido: 14/06/2011

Revisado: 12/09/2011

Aceptado: 28/11/2011

Disponible on line: 15/02/2012

\section{Resumen}

El presente artículo examina distintas experiencias de observación participante dentro del método de Grupo operativo en la intervención social y en la docencia universitaria. De ellas se pretende extraer claves metodológicas que permitan desarrollar un estilo profesional de Trabajo Social emancipador, esto es, que comprenda y maneje el poder en la relación profesional oponiéndose a un estilo de control. El texto se centra en el análisis teórico y empírico del grupo operativo y los métodos participativos, y su relación con el poder y el aprendizaje. Más que a nivel socio-político, el presente estudio hace hincapié en las prácticas que las/los profesionales desarrollan desde sus respectivos roles de trabajo, ya sea en los Servicios Sociales o en la enseñanza superior. Se destacan como claves metodológicas: el reconocimiento de las distintas subjetividades, la necesidad de una intervención de tipo colectivo, la reflexividad metodológica, el uso de espacios de encuentro y reflexión, el trabajo teórico, el aprendizaje de tipo afectivo, la supervisión profesional, la promoción de relaciones horizontales y el esfuerzo por promover la vinculación y el desarrollo de redes.

Palabras clave: grupos, observación participante, métodos participativos, poder, docencia, emancipación.

\begin{abstract}
This article examines several experiences of participant observation within the Operating Group Method in social intervention and in university teaching. From these, we intend to extract methodological keys that allow development of a professional style of emancipatory social work, i.e., one that understands and handles power in the professional relationship as opposed to a style of control. The text focuses on the theoretical and empirical analysis of the Operating Group and participatory methods and their relationship with power and learning, not so much at the socio-structural level, but rather, focusing on practices that professional practitioners develop from their respective roles in work in social services or in higher education. As methodological keys, the following stand out: the recognition of different subjectivities, the need for a collective type of intervention, methodological reflexivity, use of spaces for meeting and reflection, theoretical work, affective learning, professional supervision, encouragement of horizontal relationships and the effort to encourage linkages and the development of networks.
\end{abstract}

Key words: Groups, Participant observation, Participative methods, Power, Teaching.

Referencia normalizada: García Martín, L. (2012): «El grupo operativo como método participativo: poder y aprendizaje en la relación profesional». Cuadernos de Trabajo Social, 25(1): 205-219.

Sumario: Introducción. 1. Marco teórico y método. 2. El método en la práctica. 3. Estilo emancipador: claves para el manejo del poder en la relación profesional. 4. Conclusiones. 5. Referencias bibliográficas.

\section{Introducción}

El poder en la práctica del Trabajo Social es un aspecto de la profesión que siempre ha estado presente. Sin embargo, la atención que el pro- blema del poder ha recibido en el seno de la disciplina ha sido variable e insuficiente. La dimensión política ha sido contemplada, tanto desde la tradición funcionalista del Trabajo So- 
cial, por su apología de la reforma social, como desde su perspectiva crítica, el movimiento latinoamericano de la reconceptualización, por promover una transformación revolucionaria de la sociedad mediante la articulación de la teoría marxista y la pedagogía de la liberación. Ambas perspectivas presentaban serias contradicciones. La funcionalista, por la reproducción acrítica de las desigualdades, mediante su discurso de normalización y adaptación. La revolucionaria, por la conjunción incompatible entre profesión y militancia ideológico-política.

En la actualidad nos encontramos frente a lo que Xavier Pelegrí denomina «una fase de amnesia en la conciencia profesional», respecto al tema del poder (Pelegrí Viaña, 2004, p. 22), que en su opinión se debe a la identificación reduccionista entre poder y política. El poder, en su definición clásica weberiana, se refiere a la probabilidad de imponer la propia voluntad en una relación social, lo que excede, con creces, a una perspectiva estrictamente político-jurídica. En esta línea es particularmente esclarecedor el análisis desarrollado por Foucault, quien estudia profusamente el poder en toda su complejidad, como una constante de las relaciones humanas que atraviesa todo el cuerpo social en múltiples direcciones. El poder existe en todo tipo de relación social, sustentando relaciones de poder. En ellas la conducta de unos es dirigida por otros a través de distintas estrategias y tecnologías, desde posiciones sociales desiguales. El poder no es ni estático ni unívoco, no tiene un origen único ni un mando supremo, no está determinado para siempre ni depende sólo de quien domina en un momento dado.

El poder es ejercido de manera distinta especialmente en función de los grandes procesos sociales e históricos que lo contextualizan. Foucault habla de sociedades disciplinarias ${ }^{1}$ para referirse a la forma contemporánea del poder, que sustituyó a la del Antiguo Régimen y que se ejerce ahora de manera difusa y sutil, mediante instituciones disciplinarias - la escuela, la prisión, la fábrica, etc.- - discursos legitimados por la ciencia y todo un «ejército de técnicos que ha venido a relevar al verdugo» (Foucault, 2002, p. 19).

Estos técnicos tienen autoridad para juzgar lo normal o lo desviado, y corregir la conducta del otro a través de la vigilancia, la sanción normalizadora y el examen (p. 175). Los criterios de la normalidad no son propios, sino que son verdades que el poder produce para perpetuar las relaciones de poder. Una de esas figuras técnicas del poder es el trabajador social ${ }^{2}$ (p. 311). Para Pelegrí Viaña (2004) gran parte del poder de un trabajador social viene dado por la organización en la que trabaja, que lo sitúa en una posición de poder desigual con el cliente pero que a la vez limita su autonomía profesional. Este poder se manifiesta a flor de piel en la relación de ayuda, mediante multitud de prácticas y discursos, con o sin la consciencia de ello por parte del profesional. El resultado puede ser, a menudo, la imposición de criterios (diagnósticos) ajenos al propio usuario que tiendan, con o sin querer, a controlar más que a ayudar al cliente, más a reproducir una desigualdad social dada que a abordarla.

El Trabajo Social está avocado entonces a repensarse a sí mismo, a estudiar formas de intervención que no sólo nos permitan no ser profesionales que desarrollan el control sino que sepamos utilizar conscientemente nuestro poder en favor de las personas con quienes intervenimos. Se vuelve preciso intentar, entonces, convertirnos en profesionales que promuevan el empoderamiento de las personas. En otras palabras, hemos de situar el problema del poder en la búsqueda de estrategias que permitan distanciarse de actuar como profesionales de control para convertirnos en profesionales de la emancipación.

Un aspecto central que hay que tener en cuenta a la hora de intentar manejar las relacio-

\footnotetext{
${ }^{1}$ Gilles Deleuze (1999) matizó que las sociedades occidentales de finales del siglo XX transitaban hacia sociedades de control, en las que las nuevas tecnologías y las nuevas condiciones del capitalismo producen nuevas formas del poder que desbordan las instituciones disciplinarias y reconfiguran el espacio social.

${ }^{2}$ Nota aclaratoria: el presente artículo utilizará de aquí en adelante el masculino genérico para designar los roles profesionales y otros términos con el propósito de referirse a todo profesional con independencia de su sexo. La alusión reiterada a roles concretos a lo largo del artículo ha impedido que las técnicas conocidas para una escritura no sexista funcionaran sin perjudicar la comprensión y ritmo del texto.
} 
nes de poder en la acción profesional es el de los métodos de intervención social. A raíz de las críticas que el positivismo viene recibiendo dentro de las ciencias sociales, en particular, como mecanismo de poder, se han producido una serie de procedimientos de investigación en los cuales el Trabajo Social puede anclarse con suma eficacia en su búsqueda de prácticas emancipadoras. Son las llamadas metodologías participativas. En particular, la Investigación Acción Participativa (IAP), que analizaré más adelante, tiene ya cierta tradición en el Trabajo Social comunitario y presenta contundentes cualidades metodológicas a la hora de abordar las relaciones de poder.

En este contexto, el presente trabajo trae una serie de experiencias en proyectos que utilizaron la Investigación Acción Participativa - entendiendo este concepto en su versión genérica que no estricta como se desarrolla en la intervención comunitaria - dentro de los Servicios Sociales y la docencia universitaria. En ellas trabajé como observador participante mediante el método de grupo operativo. Este método es una forma específica de IAP aplicada al ámbito de los grupos pequeños, y fue utilizado como forma de intervención en los siguientes dos ámbitos: en la intervención social con grupos de mujeres migrantes en riesgo de exclusión y en la docencia universitaria con estudiantes de Trabajo Social. En el primer caso, se implementó el grupo operativo en el municipio de Torrejón de Ardoz, entre marzo de 2007 y diciembre de 2008. Este trabajo consistió en la formación de un grupo de encuentro y apoyo de mujeres inmigrantes en dos etapas distintas: un primer grupo en 2007, y su reedición en 2008. En el segundo caso, el de grupos de estudiantes, la experiencia se llevó a cabo entre 2007 y 2008 en la Universidad Complutense de Madrid, donde el grupo operativo venía aplicándose durante más de una década como método pedagógico para la asignatura de Trabajo Social con Grupos. El rol principal que desempeñé en ambos proyectos fue el de observador participante de los grupos, pero también como apoyo en su coordinación.
Como antecedente, es importante señalar que el método de grupo operativo ha sido implementado en el Trabajo Social en España, tanto en la práctica del Trabajo Social como en la enseñanza universitaria, por Teresa Zamanillo, quien supervisó los tres proyectos y coordinó los grupos de ámbito universitario. ${ }^{3}$ El primer grupo de mujeres inmigrantes en Torrejón de Ardoz, la que fue la primera de estas experiencias, ya ha sido analizado en el artículo El grupo como viaje de transformación personal y social, en el que se planteó el grupo operativo como una forma de Trabajo Social integral, esto es, que aúna lo individual, lo grupal y lo comunitario. Comprobamos que dicho método resultaba muy indicado para la creación de vínculos, el desarrollo de redes sociales y la construcción de espacios de encuentro y reflexión entre sus participantes (Martín Giner y García Martín, 2008). En este trabajo, utilizo las observaciones realizadas durante las dos etapas del grupo de mujeres inmigrantes en Torrejón de Ardoz, así como la experiencia como observador en la Universidad Complutense de Madrid.

El propósito final de este artículo es el de explorar las claves que hacen del grupo operativo y otros métodos participativos una vía posible para realizar un Trabajo Social que maneje el poder dentro de la relación profesional en favor de los usuarios. El contexto específico analizado es la relación profesional, la relación de ayuda, donde el trabajador social tiene mayor dominio de sus actuaciones. Queda fuera, por tanto, un análisis que aborde el contexto político o la influencia de las organizaciones. Se propone tomar conciencia de nuestra posición de poder, delegada por el orden establecido, que conforma las relaciones no igualitarias que guardamos con los usuarios, y a partir de aquí, actuar en consecuencia, decidir en medida de lo posible qué métodos y técnicas vamos a utilizar. Aquí se propone el uso reflexivo de métodos participativos, cuya clave reside en el intento de ser consecuente con una concepción de las personas como sujetos de poder, con derecho a decidir sobre sus propias vidas, por oposición a concebirlas como objetos de poder que han de ser

${ }^{3}$ Léase la tesis doctoral Teoría y práctica del aprendizaje por interacción en grupos pequeños para una descripción del método y sus resultados en el ámbito universitario (Zamanillo, 2001), así como su aplicación en la intervención social con personas sin hogar (Zamanillo y Kochen, 2000). 
adaptados al orden establecido. De esta forma se trabaja con los sujetos y no frente o para ellos; proponemos, en definitiva, un Trabajo Social que empodere, que sea emancipador.

\section{Marco teórico y método}

\subsection{La investigación acción participativa}

La Investigación Acción Participativa (IAP) es un método de investigación social que incluye como parte activa y decisoria a los propios informantes (usuarios) en el proceso de investigación, con el objetivo de generar, en cooperación con el profesional investigador, el conocimiento necesario para modificar la realidad en favor de dichos participantes. Son la comunidad o el grupo quienes deciden cómo formular el problema que será investigado, en qué forma se procederá y qué tipo de acciones se llevarán a cabo, mientras, el investigador funciona, sobre todo, como un «organizador de la discusión, un facilitador y una persona técnica a quien puede acudirse para consultar» (Park, 2006, p.133).

La IAP constituye una forma comprometida de investigación y de intervención social que se opone a las pretensiones de objetividad propias del positivismo. La neutralidad y la presunción del objeto de conocimiento como algo separado y diferente del sujeto son sustituidas por la observación participante, esto es, la investigación que involucra al observador en la interacción social directa con los informantes, dentro de la cual se producen sistemáticamente los datos. La subjetividad del profesional no es evitada ni obviada, sino reconocida, comprendida y reflexionada.

Según Jesús Ibáñez, las ciencias sociales se enfrentan a la paradoja de la autorreferencia. En la investigación social, el sujeto y el objeto coinciden, de manera que resulta ser la propia sociedad la que intenta describirse a sí misma con los instrumentos científicos que ella misma ha creado, así como es el lenguaje el que habla de sí mismo y el investigador lo mismo que lo investigado. La relación epistemológica es, además, dinámica: es la presencia investigadora lo primero que transforma lo observa- do. Ante este panorama, el conocimiento sólo puede acercarse a cierta objetividad si la investigación es guiada por la reflexividad, es decir, si está sometida a la reflexión y a la crítica por parte de los propios investigadores y por parte de los propios informantes (Ibáñez, 1994). Una mayor objetividad sólo es garantizada por los «espacios reservados para la interrogación, la autocrítica y la heterocrítica» (Zamanillo, 2008, p. 109). La mutua transformación, originada en el diálogo, entre informante y profesional, así como la producción cooperativa de conocimiento crítico, reflexivo con vistas a actuar, hace que la investigación suponga necesariamente acción.

\subsection{El Grupo operativo}

El grupo operativo es un método de aprendizaje diseñado por el psicólogo Pichón Rivière, que aplica la IAP en el marco de los grupos pequeños. Es necesario aclarar que no se trata de un método especializado de grupos sino de un método cuyo núcleo fundamental es la reflexiónacción-reflexión en grupo, para reconocer las creencias o los estereotipos que inundan nuestras representaciones mentales y rediseñarlos de acuerdo a la realidad en la que se vive, con el fin de lograr una forma de acción coherente con la misma. Este método puede ser aplicado en cualquier ámbito y con cualquier objeto de estudio o «tópico», esto es: para aprender el proceso de los grupos, para integrarse en una realidad social diferente con motivo de la inmigración, para estudiar los problemas de integración de un colectivo concreto, por ejemplo, personas sin hogar, para trabajar con las familias, etcétera.

Los integrantes de los grupos tienen la tarea de reflexionar en el diálogo sobre las dificultades que surgen en las interacciones dentro del mismo grupo. Este grupo es guiado por el coordinador, en su proceso de evolución, hacia el objetivo de que sus integrantes lleguen a un «conocimiento tanto intelectual como vivencial de las relaciones interpersonales» (Zamanillo, 2002, p. 5).

Se pretende, además, ahondar en las ideas preconcebidas y desarrollar un esquema conceptual-referencial operativo ${ }^{4}$ de grupo, así co-

${ }^{4}$ El Esquema Conceptual Referencial Operativo (ECRO) es el conjunto de conocimientos compartidos que el grupo construye en su proceso de evolución y que constituye el marco de referencia para planificar su acción en el mundo. 
mo el esclarecimiento de las ansiedades que en el seno del grupo dificultan el aprendizaje (2008, p. 117).

El cometido fundamental del método es promover el desarrollo de sujetos reflexivos. Este concepto puede ser manejado con cierta ambigüedad, pero siempre será resaltado el papel crucial de la reflexión crítica en las acciones del individuo. Se entiende por sujetos reflexivos, en cualquier caso, aquellas personas que desarrollan la capacidad, inherente a todo ser humano, de interrogar al mundo y a sí mismas, de actuar con conciencia más que por inercia, y de integrar el conocimiento en la práctica para desarrollar formas de vinculación y acción que promuevan su emancipación y autonomía. La emancipación se entiende aquí como el proceso por el cual las personas se liberan de la alienación, esto es, de las condiciones sociales o psíquicas que impiden su desarrollo y realización personal. La alienación puede ser sinónimo de situaciones de dependencia, es decir, de la incapacidad para conseguir autonomía personal. Teresa Zamanillo desarrolla un debate en torno a este tema revisando los postulados de la teoría crítica; concluye que los objetivos emancipadores de la profesión del Trabajo Social han de centrarse en los procesos de diferenciación del sí mismo ${ }^{5}$ (2002:31) y en la movilización de poder subjetivo: «la superación de una actitud pasiva de víctimas» (p. 137) para tomar conciencia de la propia capacidad de operar en el entorno y modificarlo. Tanto la diferenciación como la movilización del poder pueden ser entendidas como desarrollos de la autonomía. Una persona con un alto grado de diferenciación del sí mismo es una persona autónoma, porque sus sentimientos y acciones no dependen directamente de los de otros, en el sentido de que puede decidir contrariarlos o seguirlos sin sufrir un alto nivel de ansiedad. Así, también, una persona que aumenta su poder subjetivo, su poder de hacer, su poder para obrar, está caminando hacia su propia autonomía, en el sentido de que es menos dependiente, o está menos sujeta, de anclajes que impiden su acción y su autorrealización. En este aspecto, ha de tenerse en cuenta que la autonomía posee una dimensión colectiva inalienable. Tanto la maduración personal del individuo como su emancipación de condiciones alienantes requieren la diferenciación con el otro, respecto al otro, o la vinculación con los otros para poder afectar en problemas supraindividuales.

El método encara sus objetivos a partir de un soporte teórico fundamentado en una perspectiva sociopsicológica (Zamanillo, 2008, pp. 20-52). Se conciben las relaciones individuosociedad como un todo relacional, dialéctico, dinámico, construido en la interacción, y se utiliza, para ello, la teoría psicoanalítica, el interaccionismo simbólico, la teoría crítica marxista y la teoría sistémica (pp. 54-81).

La puesta en práctica del método se llevó a cabo, en las experiencias que analizaré, por el equipo de coordinación, el cual orienta su intervención con el grupo desde distintos espacios de reflexión. El equipo está integrado por los roles de coordinador de grupo, observador participante y supervisor. Coordinador y observador intervienen directamente en las sesiones grupales, las cuales, a menudo, se realizan semanalmente. Tras las sesiones, o cuando por necesidad se requiera, las figuras de coordinador y observador realizan reuniones de equipo para hablar de lo acontecido en el grupo. Entre tanto, se realizan las reuniones de supervisión, que incluyen también al supervisor, y que pueden efectuarse cada dos semanas. En ellas se reflexiona sobre el devenir del grupo y sobre las relaciones equipo-grupo, analizando la transferencia y la contratransferencia ${ }^{6}$, y planificando la acción profesional siempre en función del estado evolutivo del grupo y de las necesidades de sus integrantes.

\footnotetext{
${ }^{5}$ Murray Bowen define la diferenciación del sí mismo como el «proceso a largo plazo por el que el hijo se desvincula de la fusión inicial con la madre y se mueve hacia su propia autonomía emocional» (Bowen, M., 2009:70). Este proceso hacia la autonomía emocional se refleja y se desarrolla en todas las relaciones interpersonales del individuo.

${ }^{6}$ La transferencia y la contratransferencia son términos psicoanalíticos que designan las emociones que se despiertan en la relación profesional como resultado de las resonancias que el otro provoca en nuestro inconsciente. La transferencia corresponde a lo que el usuario siente hacia el profesional y la contratransferencia se refiere a los sentimientos que se dan en la dirección inversa.
} 
Las funciones generales de cada figura del equipo son las siguientes: el coordinador acompaña al grupo en su evolución, lo reorienta en su tarea, y realiza devoluciones, esto es, sistematizaciones y puntos de vista a partir del discurso del otro, procurando hacerlo siempre después de comprender al grupo; el observador registra datos y los interpreta para gestar hipótesis que puedan apoyar la labor de coordinación; y el supervisor, portador de mayor experiencia que los otros profesionales, interpreta en profundidad las relaciones en el grupo y entre el grupo y el equipo. Las tres figuras se acercan a la realidad utilizando la observación participante.

Tanto grupo como equipo de coordinación están produciendo conocimiento en todos los espacios de reflexión. El conocimiento no se concibe aquí como una información de carácter objetivo que se pueda transmitir, sino como algo que se construye en la interacción, en un marco de intersubjetividad ${ }^{7}$. Zamanillo se refiere a este aspecto de la siguiente manera:

Se persigue con ello romper con el esquema clásico de la investigación en la que el observador se sitúa, de acuerdo con un paradigma de control, en el lugar del saber, de la certeza y, por tanto, el del poder; es el lugar desde el que describe, analiza, prescribe y diagnostica situaciones, y dicta conductas. Se pretende también el entrenamiento en las reglas metodológicas de la observación; y, cómo no, lo más importante, se busca fomentar la reflexión de los sujetos, lo que les guiará y ayudará a establecer conductas de un modo consciente, con criterios propios y con una diferenciación personal que se crea y recrea en la intersubjetividad; se busca que vayan haciéndose, en una palabra, sujetos reflexi$\operatorname{vos}(2002$, p.81).

El conocimiento tampoco ha de entenderse en su sentido clásico, como una función meramente cognitiva. En el aprendizaje en grupos, continuamente se están movilizando emociones, se están experimentando tensiones, ansiedades, placer, y se están trabajando distintos modos de relacionarse. El aprendizaje, por así decirlo, pasa por el cuerpo, y resuena, además, en nuestras biografías, en nuestros recuerdos. El conocimiento es, entonces, algo más complejo, que abarca y relaciona el sentir, el pensar y el actuar. La reflexión produce lo que David Ausubel llama aprendizaje significativo: el nuevo conocimiento es un producto de la interacción entre las nuevas ideas y las que ya se sabía, de manera que una información es comprendida en la medida que se relacione lo ya aprendido y lo nuevo de forma no arbitraria y no literal (Ausubel, 2009, pp.121-168). En la aplicación docente del método se hace particular énfasis en este tipo de trabajo reflexivo con los contenidos teóricos. No obstante, la clave del aprendizaje en los grupos pequeños hay que situarla en lo que se refiere a la experimentación de relaciones interpersonales. Los conflictos y las tensiones que aparecen en los grupos aprenden a ser manejados por sus participantes, como no podría ser de otra forma, mediante la práctica, lo que genera un precedente de maduración personal aplicable en la vida cotidiana.

\section{El método en la práctica}

A continuación se describen las experiencias de grupo operativo antes nombradas. Primero expongo, desde el plano subjetivo, cómo se ejerció la labor de observador participante, cuáles fueron mis ansiedades y mis herramientas, con la intención de generar una comprensión lo más íntima posible de lo que supone desempeñar este rol. Después, describo las experiencias de los distintos grupos mencionados en términos de proceso y resultados, para proveer una idea general de cómo funciona el método en la práctica y para presentar datos que permitan evaluar el carácter emancipador de estas prácticas.

\subsection{La observación participante en el Gru- po operativo}

La observación participante, entendida como una técnica, es común al coordinador y al observador de grupo operativo. Sin embargo, co-

\footnotetext{
${ }^{7}$ Se entiende aquí por intersubjetividad «la relación establecida entre varios seres con el objetivo de llegar al conocimiento, y cuyas características principales se reducen a la comparecencia actual de los interlocutores, a la proximidad, y la permuta de opiniones entre los sujetos. Lo intersubjetivo apela a la disposición del yo a los otros, a la interacción mutua, donde la interdependencia gobierna los lazos rectores de los hombres, de los grupos y de sus actividades» (López Cruchet, 1995, pp. 749-750)
} 
mo rol específico, el observador se diferencia del coordinador en que el primero no ha de hacer devoluciones ni interpretaciones al grupo durante las sesiones. Mientras que el coordinador interviene verbalmente a lo largo de la sesión para apoyar al grupo en su tarea, el observador produce datos que serán utilizados fuera de la sesión o, en todo caso, en una devolución al final de ésta.

Desde el punto de vista particular de mi experiencia, la observación participante funciona de la siguiente manera. Lo primero es, obviamente, observar. Sentado en el círculo de sillas en el que el grupo conversaba, mi labor trataba fundamentalmente de escuchar. Escuchar significa, en este caso, poner atención en los cinco sentidos y en la intuición, en busca de toda aquella información que permita construir hipótesis sobre lo que ocurre en el grupo. Se trata de hacer un esfuerzo por concentrarse que nos lleve a un estado de alerta en dos direcciones. La primera, prestando atención al grupo: a las palabras y a los cuerpos, al lenguaje verbal y al no verbal, a lo que se dice de forma explícita y a los signos que hagan intuir un discurso implícito, y a todas aquellas interacciones que evidencien el estado evolutivo que atraviesa el grupo. Será la teoría, la experiencia y la curiosidad las que determinen hacia dónde se dirige nuestra atención ya que, obviamente, es imposible observarlo todo. Y como segunda dirección, la atención se dirige hacia uno mismo. El observador reacciona siempre a lo que acontece en el grupo pues está implicado en el sistema: las palabras pueden resonar en él provocando emociones de distinto signo. El observador está sintiendo en cada momento, por ello, parte de la atención ha de situarse en vigilar las propias entrañas. El completo silencio que ha de guardar a lo largo de la sesión, norma fundamental de este rol en el método de grupo operativo, es un asunto que acaba por ser frustrante. El observador lo escucha todo pero no puede opinar, y si se siente atacado, no puede «defenderse». Si al final de la sesión realiza devoluciones al grupo, tiene que ser lo suficientemente consciente para que sus reacciones emocionales no impregnen lo que va a decir.
Más allá de las reacciones ante la comunicación en el grupo, el observador puede reaccionar también ante la propia situación de observador. En mi caso, estuvo presente, a menudo, el miedo al rechazo. La posición de «mirón», en la cual uno lo oye todo y puede no decir nada, produce la temida fantasía de una rebelión del grupo contra este sujeto callado, que se dedica a tomar notas mientras los demás comparten su intimidad. El miedo adquiere diferentes formas según la composición del grupo. En mi caso, experimenté ansiedad por motivos diferentes según el tipo de grupo. En los grupos de intervención social, estaba presente la idea de que todas las participantes eran mujeres y yo el único hombre, en los grupos de la Universidad, en los que todos eran estudiantes, jóvenes mujeres y hombres, mis iguales, tenía la sensación de tener que estar legitimando un estatus diferente respecto a ellos dentro de la clase.

Las observaciones son registradas en un cuaderno durante las sesiones. También se graban las conversaciones y se transcriben. A partir de estos materiales la labor del observador es describir la comunicación en el grupo e interpretarla, esto es, elaborar hipótesis acerca de las fuerzas $^{8}$ que operan en el grupo y de su estado evolutivo. Tanto la observación como la interpretación son posibles gracias a la formación teórica. Las interpretaciones consisten en cadenas argumentales, mediante herramientas conceptuales, acerca de lo percibido. Como se dijo más arriba, las líneas fundamentales vienen del interaccionismo simbólico, la teoría de sistemas, la teoría crítica y la psicodinámica. Es obvio que mi formación en cualquiera de esas perspectivas era superficial dado que acababa de terminar la diplomatura. En cualquier caso, existen una serie de claves fundamentales que no necesitan de un largo recorrido de formación teórica pero sí de cierto adiestramiento guiado, cómo no, por la coordinadora, la supervisora y las lecturas, elementos todos que facilitan el aprendizaje a través de este método. Destaco, como claves en las que fijarse dentro del grupo: la comunicación y el tipo de interacción, en particular si es hacia la coordinadora o entre los

${ }^{8}$ Para una explicación general de las fuerzas que actúan en los grupos, léase Dinámica de grupos: cincuenta años después de Lopez-Yarto (2001). 
participantes, los signos verbales de que haya sentimiento de pertenencia, en particular la palabra nosotros, y la dinámica de reflexión, si el grupo se centra en su tarea o es incapaz de centrarse en los temas que desarrolla.

La teoría sirve, además, para interpretar el momento grupal. El momento grupal es el estado evolutivo del grupo en un momento dado de su desarrollo, lo que conformará su capacidad para trabajar de manera autónoma hacia los objetivos fijados. Es necesario tener esto en cuenta para no exigirle al grupo tareas que no puede asumir dada su inmadurez o para no excitar situaciones de especial transferencia psicodinámica. Y es que todo grupo atraviesa una serie de etapas definidas por un tipo de comunicación diferente. Aunque la forma concreta en que un grupo se desarrolla es algo impredecible, sí es posible estipular una serie de etapas que, necesariamente, todo grupo tiende a recorrer, aunque sea sólo de forma parcial, con retrocesos y saltos.

Un modelo de desarrollo grupal muy utilizado es el de Bennis y Shepard, quienes, en términos generales, entienden la evolución de cualquier grupo como un camino de la dependencia del coordinador a la interdependencia entre los miembros, es decir, una evolución hacia la autonomía grupal. La aptitud del grupo para trabajar en equipo por sí solo crece en función de su desarrollo hacia dicha interdependencia. Es característico que los temas abordados vayan cambiando en lo relativo a la autoridad, en la fase de dependencia, a las relaciones entre iguales, en la fase de interdependencia (López-Yarto, 2001, p.177).

Otro modelo de desarrollo grupal que resulta muy útil es el de Wilfred Bion. En este caso, se parte de la teoría psicoanalítica para diferenciar entre grupo básico y grupo de tarea. Un grupo básico es un grupo que se encuentra atravesado por uno de los tres supuestos bási$\cos ^{9}$ de Bion; esto es, por un estado emocional común al grupo que funciona de manera inconsciente y que incapacita a dicho grupo para realizar su tarea. Un grupo de tarea es un grupo que no se encuentra dominado por ninguno de los supuestos básicos y puede centrarse en la tarea, o sea, en el desarrollo de la discusión, y en cooperar de forma eficaz en el trabajo común. Cualquier grupo llega a situarse en los supuestos básicos sin un orden o duración aparente entre ellos, por lo que no se trata de etapas, sino de estados transitorios y recurrentes (Bion, 1980).

\subsection{El Grupo operativo en la práctica de la intervención social}

La experiencia que expongo en este apartado se desarrolló en el municipio de Torrejón de Ardoz, en colaboración con la Universidad Complutense de Madrid, a través del proyecto «Grupos Solidarios». Se llevó a cabo en dos etapas: una primera versión a lo largo de 2007 y una reedición durante 2008. La primera etapa se concibió como un espacio de reflexión y acción para mujeres inmigrantes con un perfil de vulnerabilidad, definido por la escasez de red social. El objetivo fue la creación de un grupo de encuentro y apoyo que sirviera como servicio de asesoramiento y autoayuda, y que favoreciera la participación social de las mujeres en el municipio. Se trabajó especialmente el diálogo reflexivo y la creación de vínculos entre ellas, y de ellas con las instituciones.

La clave del proyecto residió en el requisito del discurso abierto. Una de las virtudes de estos grupos es que permiten a los propios usuarios decidir sobre qué se conversa. Gracias a ello pueden traer sus verdaderas preocupaciones, más allá de lo que el profesional estime importante. La clásica posición de poder propia de un coordinador de grupo que trae él mismo el tema y hace las preguntas queda subvertida, en este caso, ante un grupo que produce su propio discurso y que genera sus propias preguntas. Las mujeres de este grupo compartían sus problemas cotidianos en un espacio de reflexión semanal formado por personas en situaciones similares, compartidas, y por tanto,

\footnotetext{
${ }^{9}$ Los tres supuestos básicos son los siguientes: de dependencia, en el que el grupo se comporta como si el cometido de la reunión consistiera en recibir de forma pasiva la dirección del líder o coordinador, que es contemplado de manera omnipotente; de ataque-huida, en el que el grupo se comporta como si se defendiera de un enemigo real o imaginario a través de los mecanismos de ataque o huída; y el de emparejamiento, en el que el grupo funciona arropando a una pareja sexual, que es contemplada como promesa mesiánica y redentora para todo el grupo (Bion, 1980).
} 
se comprendían mutuamente en un marco de identificaciones mutuas. Es en este tipo de diálogos donde los sujetos pueden desarrollar reflexiones que les ayuden a transformar su vida cotidiana. Un ejemplo fue el replanteamiento de la vida sentimental, familiar y reproductiva de una de las participantes:

No quiero correr $(\ldots)$ ¿De qué me sirve tener un hijo y que mañana me lo vayan a quitar? Tengo que pensarlo, porque él (su pareja) viene de un fracaso y yo de tres. Si quiere un hijo, yo se lo puedo dar, porque no soy estéril, pero todo a su tiempo.

En este grupo la reflexión fue incorporada poco a poco como un hábito. Las mujeres se enfrentaban continuamente a difíciles decisiones en su vida diaria, y el grupo sirvió como un espacio donde discutir esos dilemas; un lugar en el que las mujeres se comprendían de una forma muy personal mientras la coordinadora las ayudaba a profundizar en las discusiones. Esta dinámica queda reflejada en las siguientes citas textuales de una participante:

Cuando tengo un problema voy y se lo cuento a ellas, o voy y entre todas damos la idea. O ella tiene un problema y nosotros venimos y le damos lo que ella necesita. Un apoyo, ¿entiendes?, un apoyo de familia.

Esta práctica se volvió una rutina en el sentido de que tanto la filosofía del método, como la práctica de las participantes, acabaron por estar impregnadas de la máxima «comprende y comparte antes de actuar»».

En cuanto al apoyo de la coordinadora, las intervenciones iban en diferentes líneas. Cabe destacar la intención constante de promover que las mujeres pusieran palabras a lo que sentían: «¿qué sentías ante la ausencia de las compañeras?». La coordinadora también realizaba interpretaciones acerca de lo que sucedía de forma implícita en el grupo y que lo estaba afectando:

Habéis traído dos temas principales: por un lado el tema del cotilleo, crítica de unos a otros, y luego el tema de la falta de ayuda afuera. Entonces, yo, por traeros a reflexionar, os planteo que quizás estéis hablando de esos temas porque aún no os conocéis... entonces, os estáis preguntado ¿me criticarán a mí en este grupo? ¿me ayudarán a mí en este grupo?

El método de grupo operativo, además de desarrollar las sesiones de discusión, consigue que el grupo siempre se vea complementado por el apoyo del equipo de coordinación. Primero, por el de la trabajadora social, que desempeñaba el rol de coordinadora en la propia sesión y que se ayudaba, en este caso, de la formación en perspectiva sistémica para interpretar y hacer devoluciones; así como también utilizaba el conocimiento de los recursos del municipio para facilitar información y realizar derivaciones a otros servicios. El segundo apoyo consiste, como antes quedó descrito, en la labor del equipo de coordinación y la supervisión profesional.

Bajo este sistema de trabajo, y a lo largo de 2007, el grupo fue desarrollando la integración social de las participantes mediante la vinculación entre ellas y de ellas con otros espacios. Se realizaron varias derivaciones, por ejemplo, al equipo de terapia familiar del Consejo $\mathrm{Mu}-$ nicipal de Bienestar Social o a la unidad de atención psicológica de la Concejalía de la Mujer. También se propuso participar al grupo en diferentes espacios y actividades del Municipio, como los encuentros del Banco del Tiempo o las actividades del Centro de Desarrollo Comunitario del barrio San José. Finalmente, se apoyó al grupo en su decisión de intentar constituirse como asociación, sólo que, tras explorar las distintas opciones, finalmente no se intentó.

El proyecto se reeditó en 2008. En este período, se incorporaron nuevas participantes y se modificó la estructura de las sesiones. Se intentó añadir una explicación teórica acerca de la comunicación humana antes de cada sesión grupal. En un principio se estimó que este cambio resultaría enriquecedor para las participantes pero, en la práctica, aparecieron dos nuevos problemas. Primero, que dado el limitado tiempo libre con el que contaban las mujeres, era necesario, o bien más tiempo, o bien recortar las sesiones grupales. Y segundo, que el matiz de curso-taller que introducían las exposiciones teóricas, acabó por perturbar el discurso abierto del grupo, subordinándolo, en la práctica, al contenido del curso. Ello llegó a 
afectar a la relación profesional, pues la dinámica de profesora-alumna, en la que el grupo atiende a las explicaciones de la profesora, y a la que se opone en teoría este método, tendió a reproducirse con mayor facilidad. Cabe decir que este tipo de interacción es muy común en los primeros pasos de cualquier grupo o en los grupos que se asientan en un supuesto de dependencia. Sin embargo, en este caso, no resultó ser un problema predecible, sino una dificultad añadida que hubo que afrontar desde el equipo de coordinación, porque perturbaba la evolución del grupo hacia una forma de interacción más autónoma.

Ese año, el proyecto tuvo que enfrentarse a nuevos problemas no presentes en la edición anterior. La asistencia se volvió muy escasa a mitad de año, hasta el punto de tener que reconfigurar por completo el proyecto. La ausencia de un grupo estable nos llevó a responder con una dinámica basada en talleres abiertos para un perfil de usuarios mucho más amplio: cualquier mujer con algún tipo de contacto con los Servicios Sociales. La hipótesis más importante que se manejó como diagnóstico de la situación fue que, por la reestructuración administrativa que el municipio sufrió ese año, la eficacia de los Servicios Sociales, a la hora de derivar participantes con el perfil requerido, disminuyó notablemente. La situación hizo que replanteáramos la propia esencia del proyecto. Latió la duda de si, entonces, era lo más adecuado constituir grupos estrictamente formados por mujeres inmigrantes, o si el nuevo formato con exposiciones teóricas trajo más costes que beneficios.

A pesar de todas las dificultades, de nuevo se produjeron buenos resultados. En términos de emancipación y empoderamiento hay que valorar los siguientes hechos:

- Todas las integrantes de los grupos desarrollaron su red social. Primero, por el conocimiento y vinculación con distintas organizaciones del municipio, y segundo, por la vinculación entre ellas.

- Todas las participantes ganaron una matriz de apoyo fundamental que paliaba la ausencia de la familia de origen en España.

- Todas las participantes aumentaron su posición de poder dentro de la vida social en el municipio, debido al contacto con asociacio- nes y a la posibilidad de interaccionar con las administraciones como grupo, no solamente como individuos, y ante la posibilidad de constituirse en colectivo u asociación.

- Todas las participantes experimentaron con asiduidad, e incorporaron como un hábito en sus vidas, la reflexión dialógica, llegando a poner en duda sus propias nociones y prejuicios. En particular, fueron recurrentes en estos grupos los temas del racismo y las relaciones de género.

\subsection{El Grupo operativo en la práctica de la docencia universitaria}

El curso 2007/2008 fue el último de los más de diez años de utilización del grupo operativo como método pedagógico en la asignatura de Trabajo Social con grupos en la Universidad Complutense Madrid. En este proceso, la versión del método incorporaba la presencia obligada de determinados temas y textos a discutir, en parte de las sesiones, así como la alternancia con clases lectivas semanales bajo el paraguas clásico de la explicación teórica por parte de la profesora. El discurso abierto era, pues, una condición de sólo una parte - la mayoría- de las sesiones. Aun así, las intervenciones de la coordinadora — papel desempeñado por la profesora - nunca iban dirigidas a controlar el conocimiento adquirido en las lecturas, o a preguntar con la intención de que los alumnos llegaran a conclusiones que la profesora ya tenía en su cabeza, sino que, siempre y únicamente, la coordinadora hacía sus aportaciones sobre el discurso que producía el grupo, y siempre en la línea de profundizar en las propias reflexiones de éste.

En este caso, y como novedad respecto a los cursos anteriores, no se trataba de un mismo grupo con seguimiento semanal, como se narra en la tesis de Teresa Zamanillo, sino a la alternancia de distintos grupos que se reunían por su cuenta, grababan y analizaban sus propias sesiones, pero que, eventualmente, y en turnos cada semana, realizaban una sesión grupal con la coordinadora y el observador, dentro de la clase y ante sus compañeros. Los estudiantes eran constantemente estimulados para desarrollar la observación sobre su propio grupo o sobre el de los compañeros. Al final de las clases se comentaban en conjunto las distintas observaciones. Fuera de las aulas, 
los grupos eran supervisados en reuniones con la profesora y el observador participante. Las dificultades surgidas dentro de los grupos eran analizadas por los propios alumnos con el apoyo de la profesora y el observador. Estos grupos de estudiantes tenían el cometido de elaborar un dossier a final de curso que sirviera como testigo de todo su proceso de grupo y como medio para trabajar el contenido esencial de la asignatura.

Para la formación en Trabajo Social con grupos la eficacia del método reside en que los estudiantes aprenden a observar los fenómenos grupales y a comprenderlos y experimentarlos en primera persona. Esta base es necesaria para cualquier intervención profesional posterior en grupos, pues no es posible hacer un trabajo con un mínimo de eficacia sin tener en cuenta los complejos procesos que hay detrás de cada grupo. Los estudiantes que pasan por estos procesos de aprendizaje notan sus propios cambios a la hora de observar y comprender los grupos, pero también toman conciencia de su propio comportamiento dentro de las sesiones. Es inusual el método de enseñanza que permite vernos a nosotros mismos en nuestra interacción con el mundo, no obstante, todo el que pasa por el grupo operativo en la universidad recibe el feedback de la grabadora y de sus propios compañeros. El cambio en el aprendizaje es comprobable a través de las propias narraciones que los estudiantes hacen en sus trabajos de final de curso. Un grupo de alumnos explicaba así las primeras sesiones:

En un principio no veíamos la finalidad de las sesiones, tratábamos temas insulsos y sin profundidad; no nos dejábamos hablar y, en vez de argumentar nuestras posiciones, tratábamos de convencer al otro de las mismas.

Sin embargo, el estudiante va tomando conciencia de las fuerzas que influyen en su comportamiento. Y primeramente lo hace a raíz de que siente, experimenta, dichas fuerzas.

Por el simple hecho de sentirnos observados, modificamos nuestra conducta con el fin de cumplir las expectativas que tienen de nosotros (...) A lo largo de las sesiones, la ansiedad y la frustración desaparecieron. Tratamos temas con más libertad y transparencia, trayendo la experiencia al grupo.
A final de curso los grupos pudieron interpretar su propia conducta con bastante finura, y tomaron conciencia de cómo resolvieron asuntos tan cruciales en la vida cotidiana como son los conflictos. Dos grupos diferentes escribían los siguientes párrafos:

Cuando hubo conflicto con uno de los miembros, no supimos afrontarlo, por el miedo que normalmente se tiene a asumir situaciones tensas, problemas, pero también por el hecho de que al estar más unidos, al apreciarnos como personas, (...) nos daba más reparo poner sobre la mesa los problemas intragrupales (...) Antepusimos la huída del conflicto individual a afrontar el conflicto grupal. Con lo cual, la amistad es un arma de doble filo, puede crear mayor confianza y seguridad entre los miembros pero también puede provocar una huída del conflicto por temor a que el vínculo amistoso se rompa.

Hemos aprendido a trabajar en grupo. Eso no significa que no hayamos tenido problemas, todo lo contrario hemos aprendido a trabajar debido a todos los conflictos que hemos vivido (...) Esto se ha visto reflejado a la hora de debatir, pasando de una fase en la que todos exponíamos claramente nuestro discurso sin tener en cuenta al resto, a una fase en la que se realiza un vaivén entre la escucha del discurso y el decirlo.

La profesora-coordinadora juega un papel crucial en estos procesos de aprendizaje, sin embargo, su importancia queda definida, precisamente, por intervenir en los grupos única y estrictamente cuando sea necesario. Una conclusión que extraigo de estos cursos es que sólo merece la pena hacer apuntes, preguntas y demás intervenciones verbales cuando se ha escuchado concienzudamente al grupo y se comprende qué está pasando dentro de él. Si no es así, simplemente, lo mejor es callar. En esta experiencia, en concreto, las intervenciones de la coordinadora iban a menudo en la línea de incitar a los miembros del grupo a reflexionar las cosas en uno mismo, es decir, a pensarnos como partícipes del mundo del que hablamos y no objetivar la realidad fuera de nosotros como algo ajeno y estático. La expresión que a menudo surgía en los grupos y que era señalada por la coordinadora era «la gente», como sujeto. «La gente» es un uso del lenguaje que excluye al sujeto pensante de la realidad que describe y que le impide verse como partícipe de ella. Median- 
te esta expresión proyectamos a menudo lo que no nos gusta de nosotros para ponerlo en los otros. Los grupos sirvieron para reflexionar desde el punto de vista, no de «la gente», sino de «nosotros» como agentes del mundo. Las cualidades emancipadoras de esta experiencia hay que buscarlas en el rico proceso de aprendizaje en lo relativo a las relaciones humanas y en el tipo de reflexiones que acabo de señalar: las reflexiones que hacemos desde el punto de vista de sujetos responsables, que nos ayudan a sobreponernos a las actitudes de víctimas del mundo.

\section{Estilo emancipador: claves para el mane- jo del poder en la relación profesional}

Comenzaba este artículo advirtiendo los peligros que el Trabajo Social alberga como parte integrante de los mecanismos de poder que operan en la sociedad. Se planteaba la necesidad de encontrar estrategias que permitan pasar de un estilo profesional de control a uno de la emancipación. Considero que los métodos participativos que pude ver en la práctica y que se han tratado de resumir aquí contienen claves importantes que permiten lograr que una intervención social tenga dicho carácter emancipador. Desde mi punto de vista, la clave de estos métodos reside principalmente en dos aspectos:

- la relación epistemológica ${ }^{10}$ que plantea, en la que existe plena conciencia de la subjetividad del profesional, así como se contempla al otro como a un sujeto, y en este sentido, se lo respeta como a un igual;

- y la incorporación de la dimensión colectiva en la intervención y el desarrollo de la autonomía, fomentando las relaciones horizontales y cooperativas entre los participantes.

Estos dos aspectos son fundamentales si se quiere promover la autonomía de las personas a través de la relación de ayuda o de la relación educativa. Es difícil evaluar, sin un estudio en profundidad entre las manos, la tendencia general que existe en España respecto a estos dos puntos metodológicos, ya sea en las universi- dades o en los Servicios Sociales. No obstante, por lo que a mi experiencia respecta, lo más común es que se opere en sentido opuesto en ambos campos.

En el plano educativo, a pesar de la gran expectación que ha levantado la reciente reforma universitaria a nivel europeo, no existen líneas pedagógicas explícitas, acuerdos generales, o cualquier otro signo, que garanticen que las universidades vayan a abandonar lo que Paolo Freire llamaba la «educación bancaria». En este tipo de educación se manifiesta una relación de poder asimétrica entre educador y educando que reproduce los mecanismos de dominación dentro de la sociedad. Ello sucede porque de antemano se concibe que «el educador es quien sabe, los educandos quienes no saben»; «el educador es quien habla, los educandos quienes escuchan dócilmente»; «el educador es quien escoge el contenido programático, los educandos, a quienes jamás se escucha, se acomodan a él»; finalmente, «el educador es el sujeto del proceso, los educandos meros objetos» (Freire, P., 1976:74). Este tipo de dinámicas se hacen patentes en los tradicionales monólogos profesorales, destinados a producir apuntes sin excesivo criterio y que han sido el método predilecto prácticamente hasta el momento presente. No obstante, las dinámicas de la «educación bancaria» siguen a menudo presentes, aunque de una forma menos obvia, en los intentos de educar de manera alternativa; por ejemplo, cuando los trabajos en grupo se usan tan solo para resumir una información que es determinada por el profesor, o bien, cuando en los debates de las clases, quien formula las preguntas, o las responde, es únicamente el profesor. Estos comportamientos evidencian una relación fundada sobre un supuesto conocimiento objetivo que el profesor posee y transmite, no que el alumnado construya activamente. El estudiante, en cualquier caso, queda a merced de un currículum de contenidos cerrado que no responde a su propia curiosidad ni fomenta tampoco que busque respuestas a sus propias preguntas; queda atrapado, en cambio, en una contradicción a la que el docente tampoco escapa: aprender desde su propio de-

${ }^{10}$ Se entiende aquí, por relación epistemológica, la relación establecida entre sujeto y objeto de conocimiento, o más bien, entre sujetos en busca de conocimiento, ya sea en la relación de ayuda o en la relación educacional. 
seo de saber a la vez que es moldeado por otros para el desempeño de la profesión.

Sin un esfuerzo en desarrollar otras formas de relación entre profesor y alumno, difícilmente podrá esperarse que los nuevos profesionales pongan en práctica formas de relación en la intervención social que se distancien de dinámicas tipo sujeto-objeto. Esta idea de objetivación del otro, que intenta reflejar cómo se trata a veces con los usuarios, no es una abstracción difícil de comprobar sino que se manifiesta a menudo en la expresión «tienes que», por la que el profesional evidencia creerse conocedor de una solución objetiva, así como con la autoridad moral suficiente para ordenarla. Cualquier solución, por el contrario, debería ser buscada con el usuario, en un ejercicio de comprensión y cooperación entre sujetos.

Las dinámicas de control, en el sistema educativo o en los Servicios Sociales, son potenciadas, además, por una concepción mentalista del conocimiento. Señalábamos antes que los grupos sirven para un aprendizaje, no sólo intelectual, sino también vivencial de las relaciones humanas. Lo importante aquí es reconocer la dimensión afectiva del aprendizaje, que en las relaciones interpersonales acaba siendo el principal factor, y que sólo puede trabajarse a través de la práctica. Si caemos en el error de que el conocimiento necesario para un trabajador social se puede obtener, sólo y de manera consciente, mediante la lectura y el trabajo intelectual, estamos olvidando el hecho fundamental que está en juego y que nos permite estar en el mundo: nuestros cuerpos. Al fin y al cabo somos este cuerpo, y es este cuerpo quien está presente al completo en nuestras relaciones con los otros. No se puede obviar la dimensión subjetiva de ningún profesional si consideramos que es esta totalidad física y fisiológica la que siente, piensa, desea, aprende, posee irracionalidades, tiene sexo, género, clase social, procedencia, y está presente en toda relación de ayuda. La consecuencia lógica de tomar conciencia de este hecho es la necesidad de cuidar de la propia afectividad para que no afecte en perjuicio de la acción profesional. Para ello siempre es útil recibir alguna forma de supervisión experta.

Por otro lado, la dimensión colectiva y cooperativa de la educación y la intervención social es un aspecto que rara vez se cuida. La for- ma en que las instituciones públicas interaccionan con los ciudadanos, que depende en último término de los profesionales que trabajan en sus puertas de entrada, tiende a ser, a mi parecer, vertical e individualizada. En los Servicios Sociales prima la atención individualizada a multitud de personas que atraviesan circunstancias similares, que normalmente no son ayudadas por la institución a ponerse en contacto entre ellas. La relación que se fomenta, en cambio, es la que enlaza institución y ciudadano, pero no a los ciudadanos entre sí, por lo que se desemboca en relaciones verticales, asimétricas, en la que dos actores con status y posiciones de poder diferentes interaccionan, pero sin que ocurra lo mismo con aquellos que están en posiciones iguales y que, mediante la horizontalidad, podrían generar vínculos y redes de apoyo mutuo.

Por su parte, el sistema educativo, más difícil de evaluar, parece debatirse entre prácticas que fomentan, en cierto modo, las relaciones horizontales y la cooperación, como son los trabajos en grupo o los debates; y, del otro lado, prácticas que dividen al estudiantado, como son las clases en las que solamente habla el profesor y no los alumnos entre ellos, o los métodos de evaluación estrictamente individuales y competitivos, como el examen. El aprendizaje en sentido cooperativo, dialogado y reflexivo dentro de la clase, parece seguir siendo, en cualquier caso, una tendencia minoritaria que, a menudo, cuando se intenta, queda degradado por el liderazgo excesivo del profesor, que no está formado en estos métodos.

Como se ha podido comprobar en los apartados anteriores, la IAP y el grupo operativo se caracterizan, precisamente, por vincular a las personas entre sí durante los procesos de aprendizaje. Ello permite que se gesten con mayor facilidad redes de apoyo y solidaridad. El poder aumenta, también, si las personas son puestas en contacto con organizaciones y recursos locales, o si llegan a interaccionar con otros actores a nivel de grupo y no sólo individual. Si, por el contrario, en la intervención social se fomenta que las personas permanezcan aisladas entre sí, la hipótesis más razonable es que se estarán fomentando relaciones de dependencia con el Estado que no favorecen la integración de las sociedades ni la autonomía de sus ciudadanos. 
Finalmente, es preciso señalar que las virtudes y deficiencias de los métodos, técnicas, prácticas y discursos que circulan tanto en las universidades como en los Servicios Sociales han de situarse, en cualquier caso, en las contradicciones de tipo estructural que fomentan unas formas de proceder u otras; en el hecho de que ambos ámbitos pueden ser contemplados a la vez como instituciones para el aprendizaje o para el bienestar, pero también, en palabras de Foucault, como instituciones disciplinarias, bien para disciplinar los cuerpos, bien para señalar y controlar la transgresión de la norma (2002 pp.261-299). Lo relevante aquí es sostener que el uso consciente de determinados métodos de aprendizaje e intervención puede hacer de la intervención un medio para la educación, el bienestar y la emancipación, aun cuando el contexto no sea favorable. En este sentido, este artículo es una propuesta más, nacida de la experiencia y de la reflexión, de cara a reafirmar la posibilidad cotidiana de una intervención social crítica.

\section{Conclusiones}

1. El poder es inherente a las relaciones sociales y opera en el Trabajo Social de forma delegada por el orden establecido. Es necesario que el profesional de la intervención social analice y tome conciencia del poder que ejerce, con o sin quererlo, sobre otras personas y grupos, así como es preciso implementar métodos y prácticas que permitan empoderar a las personas frente a la posibilidad de dominarlas.

2. Los métodos participativos como la IAP y el grupo operativo han demostrado ser eficaces a la hora de manejar el poder en la intervención social porque tienen en cuenta la subjetividad del profesional e incorporan a los informantes como parte activa en sus procesos de cambio, con voz y criterios propios, así como incluyen la dimensión colectiva y cooperativa en pro de la autonomía de las personas, grupos y comunidades. Todo ello permite que las relaciones de poder entre profesional y usuario sean mucho más simétricas.

3. Por el contrario, si un trabajador social o un docente no reconoce ni tiene en cuenta la voz y el criterio del otro, amparándose en una supuesta objetividad en lugar de considerar la intersubjetividad como núcleo del encuentro profesional y, además, tiende a seguir aislando entre sí a los usuarios o estudiantes, en vez de fomentar las relaciones horizontales, estará controlando a los sujetos y ejerciendo su poder en detrimento de ellos, con o sin saberlo.

4. La reflexividad, como principio metodológico fundamentado en la reflexión, el análisis de las condiciones del investigador y la contemplación de los distintos puntos de vista, es una condición clave para el funcionamiento de los métodos participativos aquí estudiados.

5. La enseñanza del Trabajo Social está directamente ligada a su práctica. No puede, por tanto, esperarse que los trabajadores sociales desarrollen formas de intervención de tipo emancipador si, por el contrario, han aprendido a través de modelos pedagógicos basados en el control.

6. Los grupos operativos antes descritos, en concreto, demostraron su capacidad de empoderamiento por las siguientes claves metodológicas:

a) La observación participante, la intersubjetividad y la reflexividad frente a la presunta objetividad y falso distanciamiento que obvian las condiciones sociales y la posición social de poder del profesional.

b) La necesaria experimentación física de las relaciones interpersonales para desarrollar un aprendizaje de tipo afectivo y para poder comprender el acontecer de un grupo, rechazando un conocimiento mentalista ajeno a la práctica, a los propios sentimientos y al propio cuerpo.

c) La importancia de la teoría y la lectura para poder guiar la acción de manera crítica y coherente al contexto, así como para poder observar los fenómenos grupales y poder guiar al grupo sin perturbarlo.

d) La necesidad de supervisión profesional,

e) La necesidad del discurso abierto, que permite a las personas traer sus propios temas, preocupaciones y preguntas.

f) La reflexión dialógica como pilar de una transformación gradual y emancipadora tanto para usuarios como para profesionales

g) La vinculación de los propios informantes durante los procesos de aprendizaje y el trazado de nexos con y entre colectivos y organizaciones, el esfuerzo por tejer y desarrollar redes.

h) La necesidad ineludible de crear espacios reflexivos de diálogo en la intervención, entre los profesionales y en nuestra vida cotidiana. 
7) En definitiva, un manejo del poder en la relación de ayuda y un estilo profesional emancipador puede lograrse mediante el uso de métodos participativos $\mathrm{y}$, en general, prácticas que contemplen la voz de los otros y que fomenten su vinculación en sociedad.

Finalmente, cabe incluir una cita de Luis López-Yarto, por resumir, casual pero perfectamente, la filosofía de este artículo:
Nadie que entra en contacto con otros es neutral. Pero sólo el que hace de su falta de neutralidad una situación consciente, y convierte su sistema de convicciones en una postura bien fundada, criticada y consistente, permitirá a los que reciben su ayuda y su guía la libertad de explorar con espontaneidad sus propias actitudes, opiniones y conductas, y podrá crear las condiciones imprescindibles para que puedan aprender a aprender de sí mismos y de los demás a ser personas en relación» (López-Yarto Elizalde, 2001, p. 211).

\section{Referencias bibliográficas}

Ausubel, D. (2009). Adquisición y retención del conocimiento: una perspectiva cognitiva. Barcelona: Paidós.

Bion, W. (1980). Experiencias en grupos. Paidós. Barcelona.

Bowen, M. (2009). De la familia al individuo: la diferenciación del sí mismo en el sistema familiar. Barcelona: Paidós.

Deleuze, G. (1999). Conversaciones. Valencia: Pre-Textos.

Foucault, M. (2002). Vigilar y castigar. Buenos Aires: Siglo XXI.

Freire, P. (1976). Pedagogía del oprimido. México D.F.: Siglo XXI.

Ibáñez, Jesús (1994). El regreso del sujeto: la investigación social de segundo orden. Madrid: Siglo XXI.

López Cruchet, J. (1995). Intersubjetividad. En L. Gallino (ed.). Diccionario de sociología. Buenos Aires: Siglo XXI: 749-750.

Lopez-Yarto, L. (2001). Dinámica de grupos: cincuenta años después. Bilbao: Desclée de Brouwer

Martín Giner, A. y García Martín, L. (2008). El grupo como viaje de transformación personal y social. Cuadernos de Trabajo Social, 21, 43-61.

Park, P. (2006). Qué es la investigación acción participativa: perspectivas teóricas y metodológicas. En La investigación-acción participativa. Inicios y desarrollos, Madrid: Edit. Popular-OEI.

Pelegrí Viaña, X. (2004). El poder en el Trabajo Social: una aproximación desde Foucault». Cuadernos de Trabajo Social. 17, 21-43.

Zamanillo, T y Kochen, R. (2000). Análisis de una experiencia con grupos de personas sin hogar. Trabajo Social hoy, Revista del Colegio de Trabajadores Sociales de Madrid 29, 32-59.

Zamanillo Peral, T. (2001). Teoría y práctica del aprendizaje por interacción en grupos pequeños. www.ucm.es/BUCM/tesis/cps/ucm-t25724.pdf.

Zamanillo, T. (2008). Trabajo Social con grupos y pedagogía ciudadana. Madrid: Síntesis. 